\title{
Observation of a magic discrete family of ultrabright Si nanoparticles
}

\author{
G. Belomoin, J. Therrien, A. Smith, S. Rao, and R. Twesten \\ Department of Physics, University of Illinois at Urbana-Champaign, Urbana, Illinois 61801 \\ S. Chaieb \\ Department of Theoretical and Applied Mechanics, University of Illinois at Urbana-Champaign, \\ Urbana, Illinois 61801 \\ M. H. Nayfeh ${ }^{\text {a) }}$ \\ Department of Physics, University of Illinois at Urbana-Champaign, Urbana, Illinois 61801 \\ L. Wagner and L. Mitas \\ Department of Physics, North Carolina State University, 127 Stinson Road, Raleigh, \\ North Carolina 27695-8202
}

(Received 31 August 2001; accepted for publication 26 November 2001)

\begin{abstract}
We demonstrate that electrochemically etched, hydrogen capped $\mathrm{Si}_{n} \mathrm{H}_{x}$ clusters with $n$ larger than 20 are obtained within a family of discrete sizes. These sizes are $1.0\left(\mathrm{Si}_{29}\right), 1.67\left(\mathrm{Si}_{123}\right), 2.15,2.9$, and $3.7 \mathrm{~nm}$ in diameter. We characterize the particles via direct electron imaging, excitation and emission optical spectroscopy, and colloidal crystallization. The band gaps and emission bands are measured. The smallest four are ultrabright blue, green, yellow and red luminescent particles. The availability of discrete sizes and distinct emission in the red, green and blue (RGB) range is useful for biomedical tagging, RGB displays, and flash memories. (c) 2002 American Institute of Physics. [DOI: $10.1063 / 1.1435802]$
\end{abstract}

The area of semiconductor clusters, especially of silicon clusters, is currently one of the most active frontiers in physics and chemistry. ${ }^{1-18}$ Studies have focused on their unique structures, stability, and optical and electronic and chemical reactivity, both in free space and on the surface. ${ }^{3}$ Fabricating size-, shape-, and orientation-controlled fluorescent $\mathrm{Si}$ nanoparticles in the range of $1-3 \mathrm{~nm}(30-1000$ atoms) with reproducibility could be critical to the understanding of nanostructures and would be of significant interest to the microelectronics, optoelectronics and biomedical industries. Size uniformity is important for applications that require superlattices, high quality films, or single nanoparticle-based devices, such as fluorescent imaging and tagging. Discrete sizes with distinct emission in the red, green and blue is useful for biomedical tagging and in red, green and blue (RGB) display applications. In flash memory, nanoparticles are embedded in a metal-oxide-semiconductor field effect transistor (MOSFET) as floating gates to store electric charge. Since the shift in the device's threshold depends on the particle size, a wide size distribution would wash out the operating threshold. Only recent progress towards control of the crystallography orientation has been achieved. ${ }^{12}$

It is known that self-terminated $\mathrm{Si}_{n}$ clusters ${ }^{6-8}$ with $n$ $<10$ exhibit discrete magic numbers, whereas the abundance spectrum of $\mathrm{Si}_{n}$ clusters with $n>20$ exhibits neither special features nor discrete magic numbers. ${ }^{9}$ The shape of the large ones, however, changes from prolate to more spherical in the narrow range between $n=24$ and $30 .{ }^{10}$ In the prolate regime, atoms are arranged in one shell and form triangular surface facets, while in the spherical regime, atoms are arranged in two shells. The outer shell has the topology of a fullerene

${ }^{a)}$ Electronic mail: m-nayfeh@uiuc.edu cage, and the inner shell has a high coordination number. The dissociation energy, which is strongly size dependent for smaller clusters, is a smooth function of $n$ for $n>25 .{ }^{11}$

Stable cluster configurations with hydrogen capping have recently been presented. ${ }^{4,5}$ Moreover, in some recent electrochemical etching measurements, hydrogen-capped $\mathrm{Si}$ in the 1-3 nm size regime has been shown to become highly fluorescent, exceeding the brightness of dyes such as fluorescein ${ }^{13}$ and exhibits stimulated emission ${ }^{14}$ and harmonic generation. ${ }^{15}$ In this letter, we demonstrate that, unlike uncapped $\mathrm{Si}_{n}$ particles, hydrogen-capped $\mathrm{Si}_{n} \mathrm{H}_{x}$ particles ( $n$ $>20$ ) exhibit magic discrete numbers, and that they can be classified into a family of discrete sizes that includes 1.0, $1.67,2.15,2.9$, and $3.7 \pm 0.1 \mathrm{~nm}$ diameters. Excitation spectroscopy measures for the four smallest highest unoccupied molecular orbital (HUMO) lowest unoccupied molecular orbital (LUMO) gaps of 3.44, 2.64, 2.39, and $2.11 \mathrm{eV}$, while emission spectroscopy shows that they are ultrabright UV/ blue, green, yellow, and red luminescent particles with band peaks at $\sim 410,540,570$, and $600 \mathrm{~nm}$.

We use highly catalyzed electrochemical etching in HF and $\mathrm{H}_{2} \mathrm{O}_{2}$ to disperse crystalline $\mathrm{Si}$ into ultrasmall nanoparticles. ${ }^{11,16}$ The wafer is laterally anodized while slowing being advanced into the etchant. Because HF is highly reactive with silicon oxide, $\mathrm{H}_{2} \mathrm{O}_{2}$ catalyzes the etching, producing smaller particles. Moreover, the oxidative nature of the peroxides produces high chemical and electronic quality samples. The pulverized wafer is then transferred to an ultrasound bath for brief treatment, under which the film crumbles into a colloidal suspension of ultrasmall blue particles. ${ }^{13-15}$ Larger particles are less amenable to dispersion due to stronger interconnections. We use a post-HF treatment to weaken them and then ultrasound treatment to disperse the particles. We centrifuge the mix. The resulting 

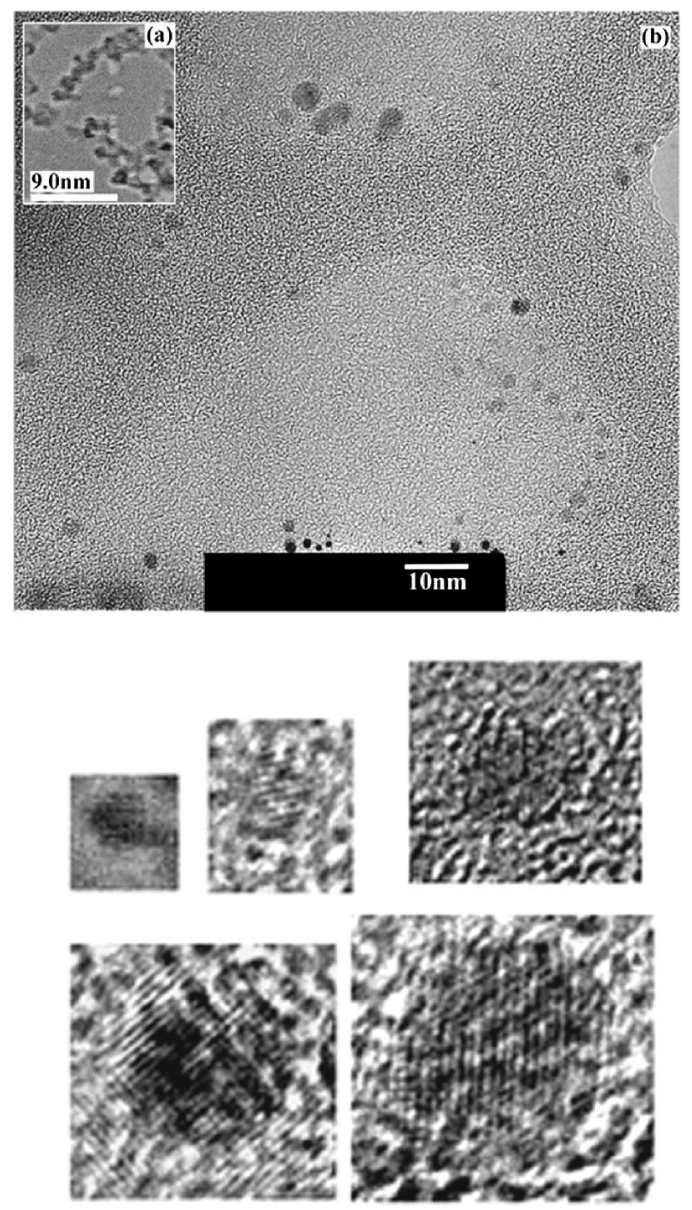

FIG. 1. (Top) (a), (b) TEM images of particles on a graphite grid. The particles are nearly spherical and can be classified into a small number of discrete sizes. (Bottom) Closeup TEM images of the 1.0, 1.67, 2.15, 2.9 and $3.7 \mathrm{~nm}$ particles.

residue consists largely of red particles, while the suspension contains green/yellow particles. We redissolve the residue and sonificate it. The red emitting particles stay in suspension. The green particles may become seperated by additonal sonification. As an alternative, we used commercial gel permeation chromatography to separate the particles. The particles are separated into four vials each containing particles of uniform size, with nearly $90 \%-100 \%$ efficiency. A thin graphite grid is immersed into the colloid and imaged by high-resolution TEM. ${ }^{17}$ Figure 1 (top) shows that the particles are nearly spherical and can be classified into a small number of sizes. They include 1.0 (see the inset), 1.67, 2.15, 2.9 , and $3.7 \mathrm{~nm}$. Figure 1 (bottom) shows closeups of the atomic planes of the 1.67, 2.15, 2.9 and $3.7 \mathrm{~nm}$ particles.

We studied the absorption and emission gaps of each member of the family. The excitation, i.e., the absorption monitored at a specific emission wavelength (the product of absorption and emission) was recorded. ${ }^{17}$ We mapped out the excitation in the range of 250 and $800 \mathrm{~nm}$, while monitoring the emission in the range of $400-700 \mathrm{~nm}$. We used mapping to identify the resonance excitation structure. Figure 2, which presents the excitation, shows resonance structures at $3.44 \pm 0.1,2.64$, and 2.39, and $2.11 \mathrm{eV}$. These resonance structures produce emission bands with maxima at 410, 540, 570 , and $600 \mathrm{~nm}$, respectively. We associate the resonance energies with the HOMO-LUMO edge $E_{g}$. According to

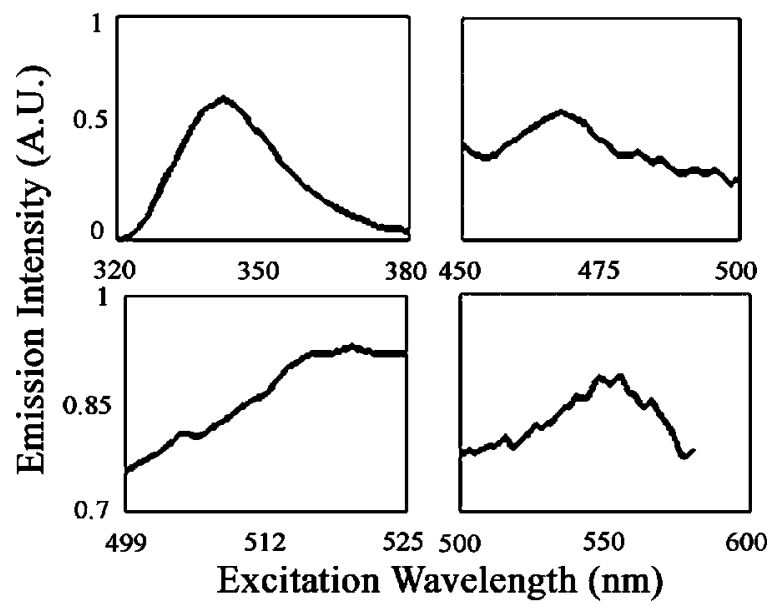

FIG. 2. Excitation spectra of the 1.0, 1.67, 2.15, and $2.9 \mathrm{~nm}$ diam particle monitored at emission wavelengths of 400, 540, 570, and $600 \mathrm{~nm}$, respectively. They show local resonance structures at 3.44, 2.64, and 2.39, and $2.11 \mathrm{eV}$, respectively.

quantum confinement, the absorption and emission photon energies correlate with the size. We pair the diameter $d$ (in $\mathrm{nm})$ with excitation resonance $E_{g}$ (in $\mathrm{eV}$ ) as follows $\left(d, E_{g}\right)$ : $(1.0,3.44)$; $(1.67,2.64)$; $(2.15,2.39)$; and $(2.9,2.11)$. However, we did not record excitation/emission resonance that can be associated with the $3.7 \mathrm{~nm}$ diam particle. This may be due to low abundance or/and diminished brightness. A power law fit gives $E_{g}=3.44 / d^{0.5}$.

Self-assembly requires size uniformity. Under certain conditions, the particles segregate according to size upon crystallization. Over time, 5-100 $\mu \mathrm{m}$ crystals have formed in a water colloid. Colloidal crystallites were placed on glass and illuminated with light from a mercury lamp (at 360, 395, $450,520,560$, and $610 \mathrm{~nm})$. Emission is detected in the reverse direction and detected by a RGB filter/prism based dispersive charge coupled device (3CCD). Figure 3 (top row) shows examples of blue, green, and red segregated crystals. Recrystallization to form yellow or green crystals takes place but they are less frequent compared to the blue and red ones. In Fig. 3 (bottom) gives a photo of colloids of magic sizes under irradiation from an incoherent low intensity commercial UV lamp at $365 \mathrm{~nm}$, showing the characteristic red, yellow, green, blue colors.

We used density functional with generalized gradient exchange-correlation potentials, configuration interaction and Monte Carlo approaches to construct structural prototype $\mathrm{Si}_{n} \mathrm{H}_{x}$ particles and to calculate the HOMO-LUMO band gap. ${ }^{5}$ We start from a spherical piece of crystalline Si. The dangling bonds are saturated with hydrogen. Pairs of $\mathrm{H}$ from adjacent surface $\mathrm{Si}$ atoms are stripped $\left(\right.$ by $\mathrm{H}_{2} \mathrm{O}_{2}$ ), and replaced by reconstructed $\mathrm{Si}-\mathrm{Si}$ dimers, similar to in wellknown Si (001) surface $2 \times 1$ reconstruction. Reconstructed $\mathrm{Si}-\mathrm{Si}$ bonds have recently been proposed as a source of novel optical activity in ultrasmall particles. ${ }^{4,18}$ The resulting structure was relaxed using density functional theory (DFT) with the PW91 exchange-correlation functional. The simulation yields stable sizes, including $1.0 \mathrm{~nm}\left(\mathrm{Si}_{29} \mathrm{H}_{24}\right)$ and 1.6 $\mathrm{nm}\left(\mathrm{Si}_{123} \mathrm{H}_{x x}\right)$, with band gaps of 3.5 and $2.67 \mathrm{eV}$, respectively. Figure 4 gives a prototype of the smallest (from Ref 5). It is $\mathrm{Si}_{29} \mathrm{H}_{24}$ with 5 atoms constituting a tetrahedral core and 24 atoms constituting a $\mathrm{H}$-terminated reconstructed $\mathrm{Si}$ AlP license or copyright; see http://apl.aip.org/apl/copyright.jsp 

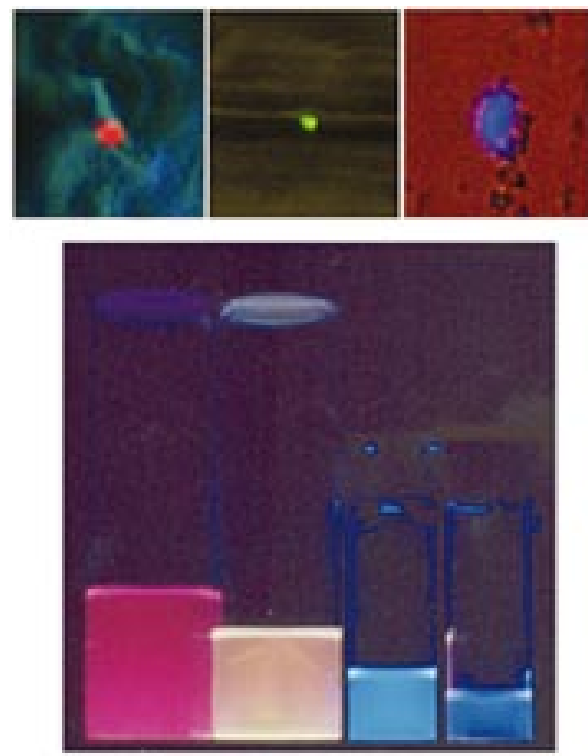

FIG. 3. (Color) (Top row) Emission from blue, green, and red colloidal crystals segregated according to magic sizes of 1.0, 1.67, and $2.9 \mathrm{~nm}$ diameters. The crystals are illuminated by light from a mercury lamp. The background is due to a weak bright field. (Bottom, right to left) Emission of colloids of four members of the magic family $1.0,1.67,2.15$, and $2.9 \mathrm{~nm}$ in diameter, after they have been separated, under excitation using a commercial low intensity UV source with an average wavelength of $365 \mathrm{~nm}$.

surface. A single $\mathrm{H}$ atom terminates each surface $\mathrm{Si}$ atom. Earlier, Allan et al. found that particles 1.03 and $1.67 \mathrm{~nm}$ in diameter are stable with band gaps of 3.5 and $2.67 \mathrm{eV}$, and consist of 29 and $123 \mathrm{Si}$ atoms, respectively. ${ }^{4}$

The dependence of the structure on the number of terminating hydrogen atoms is interesting. We start from a bulklike $\mathrm{Si}_{29}$ cluster; 36 hydrogen atoms are needed to terminate the unsaturated bonds. As the number of $\mathrm{H}$ atoms is reduced

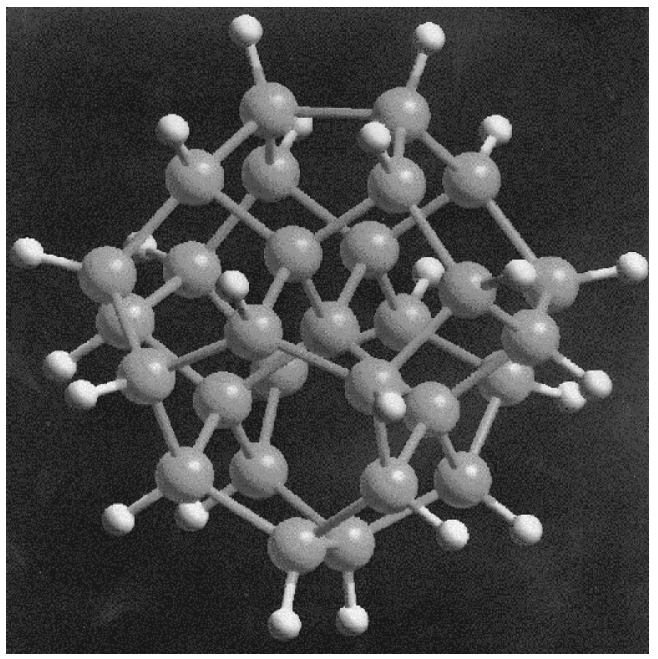

FIG. 4. Prototype structures of $\mathrm{Si}_{29} \mathrm{H}_{24}$. In the particle, $5 \mathrm{Si}$ atoms (dark circle) constitute a single tetrahedral core and $24 \mathrm{Si}$ atoms (dark circle) constitute a H-terminated (white circle) reconstructed surface. from 36, the band gap size drops slowly. But below 24, the band gap drops sharply, approaching a metallic zero band gap, and requires a bonding change from pure $s p^{3}$ tetrahedral diamond like to a mix with $s p^{2}$, a configuration common in carbon but one that does not exist in bulk Si. The particle is a filled fullurene of a highly puckered cage and belongs to TD magic structures.

The availability of discrete sizes and distinct emission in the red, green and blue range is useful for biomedical tagging, RGB displays, and flash memories. We demonstrated that $\mathrm{Si}_{n} \mathrm{H}_{x}$ clusters can be classified into a magic discrete family of spherical size that includes $\sim 1.0\left(\mathrm{Si}_{29}\right), 1.67$ $\left(\mathrm{Si}_{123}\right), 2.15,2.9$, and $3.7 \mathrm{~nm}$ diameters, emitting in the UV/ blue, green, yellow, and red for the smallest four particles.

The authors acknowledge State of Illinois Grant No. IDCCA 00-49106, U.S. DOE Grant No. DEFG02ER9645439, NIH Grant No. RR03155, U.S. NSF Grant No. BES-0118053, and the University of Illinois at UrbanaChampaign.

${ }^{1}$ Physics and Chemistry of Small Clusters, edited by P. Jena, B. Rao, and S. Khanna (Plenum, New York, 1987).

${ }^{2}$ M. F. Jarold, Science 252, 1085 (1981); K. M. Ho et al., Nature (London) 392, 582 (1998); E. Kaxiras and E. Jackson, Phys. Rev. Lett. 71, 727 (1993); J. C. Grossman and L. Mitas, ibid. 74, 1323 (1995); L. Mitas, J. Grossman, I. Stich, and J. Tobik, ibid. 84, 1479 (2000); I. Vasiliev, S. Ogut, and J. Chelikowsky, ibid. 82, 1919 (1999).

${ }^{3}$ I.-S. Hwang, M.-S Ho, and T. T. Tsong, Phys. Rev. Lett. 83, 120 (1999).

${ }^{4}$ G. Allan, C. Delerue, and M. Lannoo, Phys. Rev. Lett. 76, 2961 (1996).

${ }^{5}$ L. Mitas, J. Therrien, G. Belomoin, and M. H. Nayfeh, Appl. Phys. Lett. 78, 1918 (2001).

${ }^{6}$ W. Brown, R. Freeman, K. Raghavachar, and M. Schulter, Science 235, 860 (1987); L. Bloomfield, R. Freeman, and W. Brown, Phys. Rev. Lett. 54, 2246 (1985).

${ }^{7}$ K. Raghavachari, Phase Transitions 24-26, 61 (1990).

${ }^{8}$ U. Rothlisberger, W. Andreoni, and M. Parrinello, Phys. Rev. Lett. 72, 665 (1994); W. Andreoni and G. Pastore, Phys. Rev. B 41, 10243 (1990); U. Rothlisberger, W. Andreoni, and P. Ginnozzi, J. Chem. Phys. 96, 1248 (1992).

${ }^{9}$ L. Anderson, S. Muruyama, and R. Smalley, Chem. Phys. Lett. 176, 348 (1991); H. Kroto, J. Heath, S. O'Brian, R. Curl, and R. Smalley, Nature (London) 318, 162 (1985).

${ }^{10}$ M. Jarrold and V. Constant, Phys. Rev. Lett. 67, 2994 (1991); M. Jarrold and J. Bower, J. Chem. Phys. 96, 9180 (1992).

${ }^{11}$ M. Jarrold and E. Honea, J. Phys. Chem. 95, 9181 (1991).

${ }^{12}$ G. Grom, D. Lockwood, J. McCaffrey, H. Labbe, P. Fauchet, B. White, Jr., J. Dlenner, D. Kovalev, F. Koch, and L. Tsybeskov, Nature (London) 407, 358 (2000).

${ }^{13}$ O. Akcakir, J. Therrien, G. Belomoin, N. Barry, E. Gratton, and M. Nayfeh, Appl. Phys. Lett. 76, 1857 (2000).

${ }^{14}$ M. H. Nayfeh, N. Barry, J. Therrien, O. Akcakir, E. Gratton, and G. Belomoin, Appl. Phys. Lett. 78, 1131 (2001); M. Nayfeh, O. Akcakir, J. Therrien, Z. Yamani, N. Barry, W. Yu, and E. Gratton, ibid. 75, 4112 (1999).

${ }^{15}$ M. H. Nayfeh, O. Akcakir, G. Belomoin, N. Barry, J. Therrien, and E. Gratton, Appl. Phys. Lett. 77, 4086 (2000).

${ }^{16}$ Z. Yamani, H. Thompson, L. Abu Hassan, and M. H. Nayfeh, Appl. Phys. Lett. 70, 3404 (1997); D. Andsager, J. Hilliard, J. M. Hetrick, L. H. Abu Hassan, M. Plisch, and M. H. Nayfeh, J. Appl. Phys. 74, 4783 (1993); Z. Yamani, S. Ashhab, A. Nayfeh, and M. H. Nayfeh, ibid. 83, 3929 (1998).

${ }^{17}$ G. Belomoin, J. Therrien, and M. Nayfeh, Appl. Phys. Lett. 77, 779 (2000); J. Therrien, G. Belomoin, and M. Nayfeh, ibid. 77, 1668 (2000).

${ }^{18}$ M. Nayfeh, N. Rigakis, and Z. Yamani, Phys. Rev. B 56, 2079 (1997); Mater. Res. Soc. Symp. Proc. 486, 243 (1998). 